\title{
Research Paper: The Effect of Aquatic Balance Exercise With Weighted Vest on the Balance Recovery Strategy of Middle-Aged Women
}

\author{
Niloufar Rahmani ${ }^{\text {* }}$ (D, Amir Hossein Barati² (D, Fariborz Hovanloo ${ }^{1}$ (D) \\ 1. Department of Health and Sport Sciences, Faculty of Sport Sciences and Health, Shahid Beheshti University, Tehran, Iran. \\ 2. Department of Sport Sciences, Faculty of Sport Sciences, Shahid Rajaee Teacher Training University, Tehran, Iran.
}

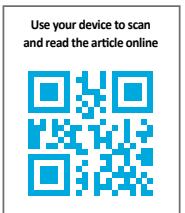

Citation Rahmani N, Barati AH, Hovanloo F. The Effect of Aquatic Balance Exercise With Weighted Vest on the Balance Recovery Strategy of Middle-Aged Women. Physical Treatments. 2018; 8(3):179-188. http://dx.doi.org/10.32598/ptj.8.3.179

doi'http://dx.doi.org/10.32598/ptj.8.3.179

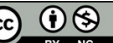

Article info:

Received: 15 Jun 2018

Accepted: 29 Aug 2018

Available Online: 01 Oct 2018

Keywords:

Hydrotherapy, Balance, Middle aged, Weighted vest, Women

\begin{abstract}
A B S T R A C T
Purpose: Disturbances in balance and also regaining the balance strategy is very important in the middle-aged women who are near their menopause and subject to severe hormonal and physiological changes, reduced muscle strength, and decreased bone density. The purpose of this study was to investigate the effect of balance training in the water with and without the weighted vest on the balance recovery strategy of middle-aged women affected with a balance disorder.
\end{abstract}

Methods: Present study was a quasi-experimental study conducted on 26 middle-aged women. They were selected by convenience sampling method and randomly divided into two groups: balance training group in water with weighted vest (13 women), and balance training group in water without weighted vest (13 people). In order to assess the balance strategy recovery, the electromyography activity of the gluteus maximus, gluteus medius, and rectus femoris muscles around the hip joint and lateral gastrocnemius, peroneus longus, and anterior tibialis muscles around the ankle joint was recorded during the drop-landing task. The water balance training was performed for 6 weeks, three sessions per week, each session for 45 minutes. After 6 weeks of training, all pretest stages were repeated in the post-test. Shapiro-Wilk and ANCOVA tests were used for analyzing the obtained data. The significance level was set at 0.05 for all tests.

Results: The Independent $t$ test results showed no significant difference between demographic information of the two groups $(\mathrm{P}<0.05)$. The results of ANCOVA showed that the recovery balance strategy of middle-aged women in the group of water balance training with weighed shirt had significantly improved $(\mathrm{P}<0.05)$. Also, the eta coefficient was 0.76 which indicates relatively large amount of impact exercises.

Conclusion: Doing balance exercises in water with weighted vest can well affect balance factors and improve the recovery balance strategy of middle-aged people. Therefore, in order to prevent disturbance in the balance and falling with increasing age, it is recommended to take advantage of water balance exercises with weighted vest in the middle-aged period.

\footnotetext{
* Corresponding Author:

Niloufar Rahmani, MSC.

Address: Department of Health and Sport Sciences, Faculty of Sport Sciences and Health, Shahid Beheshti University, Tehran, Iran. Phone: +98 (912) 2829385

E-mail:ni.rahmani@mail.sbu.ac.ir
} 


\section{Highlights}

- Balance exercise in the water with a weighted vest improves the balance and balance recovery strategies in middleaged people.

\section{Plain Language Summary}

Disruption of balance and problems in balance recovery strategies are the most important and common problems in older adults, which increases the risk of falling and injury in these individuals. Middle-aged women experience a higher risk of falling as a result of hormonal changes, menopause, and fluctuations in stature. Studies suggest that with aging, muscle's ability to use a safe and effective ankle strategy to maintain and regain balance reduces, and individuals tend to use hip strategies rather than ankle strategies to maintain their balance. This study aimed to investigate the effect of balance exercises in water with and without wearing a weighted vest on balance recovery strategy of middle-aged women. The study participants were randomly divided into two groups: one intervention group performing aquatic balance exercises with a weighted vest and one control group performing aquatic balance exercises without a weighted vest. Results showed that balance recovery strategy in middle-aged women significantly improved after performing aquatic balance exercises with a weighted vest. This intervention can enhance balance factors; hence it is recommended to take advantage of the benefits of water exercises by wearing a weighted vest at middle age.

\section{Introduction}

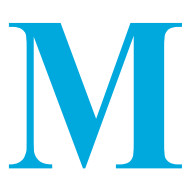

iddle age is one of the longest and most important periods of human life [1]. The last Iran population and housing census was conducted in 2016. It revealed that $44.8 \%$ of the Iranian population aged between 30-64 years (middle-aged population); $6.1 \%$ were over 65 years (old population), indicating the high percentage of the middle-aged population in the country [2]. It is also estimated that by 2031, this population will enter the old age, and the elderly will make up the majority of the Iranian population [2].

Associated problems with aging include cognitive, social, biological (health), physical and physiological changes (menopause and the end of reproductive capacity in women) [1]. It is associated with disability, physical and sexual weakness, weak physiological and psychosocial functions, muscle atrophy, reduced motion, functional disorders, strength loss, mobility loss, inability to perform daily living tasks, neuromuscular coordination deficits, degraded proprioception sense and balance, and bodily fluctuations. The above-mentioned issues may lead to functional degradation and increased vulnerability of middle-aged people [3].

Balance impairment is a subject of decreased muscle strength, increased reaction time and the proprioceptive deficit. It is among the most prevalent and important problems associated with aging and reaching middle age; it increases the risk of falling and injury in this population $[4,5]$. This is more important in middle-aged women who have undergone menopause and experienced severe hormonal and physiological changes [6]. studies have suggested that with age, women are twice as likely as men to fall because of fluctuations in stature [6] and experience its adverse consequences [7, 8]. To maintain or recover balance, humans use two strategies when faced with sudden perturbations. First, fixed-support strategy, including hip and ankle strategies; second, change-in-support strategy, including stepping or grasping movements [9].

Evidence indicated that there is an inverse relationship between the individuals' age and their balance recovery strategy; with age, they tend to use hip strategy, rather than ankle strategy to recover balance $[10,11]$. This can be due to degenerative changes in musculoskeletal and neuromuscular mechanisms, reduced muscle strength, degraded proprioception sense in the lower limbs, as well as increased reaction time [11, 12]. Based on this, middle-aged women also suffer from balance problems after undergoing menopause and hormonal and physical changes. Therefore, before the entry of this population into old age and facing aging-related problems, it is advisable to better prepare middle-aged women.

A modern training method is the use of a weighted vest and carrying additional weight during exercise [13-15]. Consequently, the use of this practice in water can have some advantages. Firstly, the weighted vest is used in a safe environment with the lowest risk of injury. Secondly, applying additional weight by wearing a weight- 
ed vest can simulate a water environment in a natural environment and reduce the effect of floating in water. Applying additional weight in water imposes the same amount of pressure and adsorbent to muscles and joints as experienced in a dry environment [16]. Therefore, this is the most efficient technique for improving physical factors, such as strength and muscular endurance, coordination, the precision of proprioception sense, and bone density [3]. Thus, aquatic exercises that can eliminate the effect of weightlessness and reduce the risk of falling for middle-aged people are useful for them. However, limited studies have explored this area.

Vanaki et al. studied the effect of aquatic aerobic exercises by applying excess weight on the spinal bone density of overweight women [17]. They reported the positive effects of these exercises on bone density. Sahebozamani et al. and Beyranvand et al. evaluated the effect of aquatic exercises on balance recovery strategy. They investigated the balance strategy of elderly men through an 8-week aquatic exercise program. They concluded that although aquatic exercise improves balance in older people, it failed to transfer the balance recovery strategy from hip to ankle after applying a sudden perturbation $[18,19]$.

Previous studies have mainly compared aquatic exercises with those in dryland environments; however, no study has compared the two aquatic exercises. Moreover aquatic exercises applying extra weight by a weighted vest were overlooked in previous studies. Moreover, it is important to know whether performing aquatic exercises while using a weighted vest can be more effective or not. Furthermore, previous studies have only examined static and dynamic balances; balance strategy as the most important variable in maintaining and the recovery of the center of gravity within the base of support has received less attention.

Beyranvand et al. explored the balance recovery strategy by the motion analyzer and using kinematic variables. However, studies have suggested that the most reliable tool for assessing the balance recovery strategy is Electromyography (EMG) device, and the study of muscle activity when faced with an external perturbation [10]. Most of the studies in Iran have examined the balance of elderly people; while middle-aged people who are subject to numerous physical changes like impaired balance [6] have been neglected. Thus, this study investigated the effect of a 6-week aquatic balance exercise program in water with and without wearing a Weighted Vest (WV) on the balance recovery strategy of middleaged women with balance impairment.

\section{Materials and Methods}

This was an applied study with a quasi-experimental design. Samples were 26 women aged 56-60 years living in Tehran City, Iran. The subjects were selected by purposeful and convenience sampling techniques. The sample size was determined using the following formula and according to previous similar studies $[9,20]$ :

$$
\begin{gathered}
\left(Z_{1}-\alpha / 2+Z_{1}-\beta\right)^{2}\left(S_{1}^{2}+S_{2}^{2}\right) /\left(M_{1}-\right. \\
\left.M_{2}\right)^{2}=1.64+1.28^{2}\left[0.8^{2}+1.1^{2}\right] / 3.5-2.4^{2} \approx 13
\end{gathered}
$$

Inclusion criteria were being female; being aged of 56-60 years; not being in the acute phase of menopause; physical health; normal Body Mass Index (BMI), no pathological symptoms, fracture, surgery and joint diseases in lower extremities; not having the shortness of breath, heart failure and cardiac palpitations; and achieving a lower limb length of $<0.94$ in the $\mathrm{Y}$ balance test (as the sign of balance impairment) [21]. Exclusion criteria were inability to perform exercises; not attending in two consecutive sessions or three alternate sessions; feeling pain in any part of the body during exercises; not participating in the post-test measures.

To ensure the health of subjects, they were requested to complete the General Health Questionnaire (GHQ) and the Physical Activity Readiness Questionnaire (PAR-Q). A written informed consent form was obtained from the study participants. Then, they were randomly divided into two groups of WV (performing aquatic balance exercises with WV) and control (performing aquatic balance exercises without WV).

After fully explaining to the participants how the test should be performed, they were requested to perform some trials of the drop-landing task [22]. Before placing electrodes, the skin area was prepared by shaving the excess hair and cleaning with cotton and medical alcohol. Then, electrodes were placed on the selected muscles according to Surface Electromyography for the Non-Invasive Assessment of Muscles (SENIAM) (European recommendations for surface electromyography) and considering the center-to-center distance of $20 \mathrm{~mm}[23,24]$.

A footswitch was installed on the subjects' toes. Then, they were requested to stand barefoot on a wooden box (height $=30 \mathrm{~cm}$ ) for performing the drop-landing task. Their test foot (dominant leg) to which electrodes were attached, must had no contact with the box and other foot could bear the subject's weight. To provide better safety 
Table 1. The protocol of aquatic balance exercises

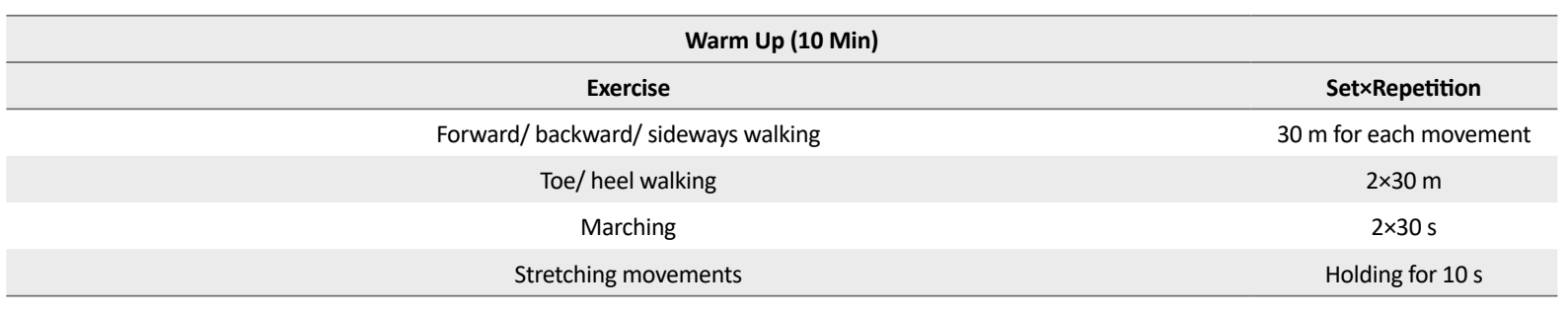

\begin{tabular}{|c|c|c|}
\hline & Balance Exercises (30 Min) & \\
\hline Week & Exercises & Set $\times$ Repetition \\
\hline \multirow{6}{*}{1} & Carnival walking & $2 \times 30 m$ \\
\hline & Spiral walking & $1 \times 30 \mathrm{~m}$ \\
\hline & Foot pump (ankle plantar flexion) & $3 \times 10 r$ \\
\hline & Both-leg squat & $3 \times 8 r$ \\
\hline & Single-leg stance & $3 \times 20 s$ \\
\hline & Circle walking (clockwise and anti-clockwise) & $3 \times 30 \mathrm{~s}$ \\
\hline \multirow{6}{*}{2} & Walking Carnival & $2 \times 30 \mathrm{~m}$ \\
\hline & Spiral walking & $2 \times 30 \mathrm{~m}$ \\
\hline & Both-leg squat and ankle pump & $3 \times 8 r$ \\
\hline & Single-leg stance with a training partner & $2 \times 20 \mathrm{~s}$ \\
\hline & $\begin{array}{l}\text { Circle walking (clockwise and anti-clockwise, while one is standing on both } \\
\text { feet in the center of circle) }\end{array}$ & $3 \times 30 \mathrm{~s}$ \\
\hline & Front-to-back weight shift & $3 \times 5 r$ \\
\hline \multirow{6}{*}{3} & Walking carnival with bending knees & $2 \times 30 \mathrm{~m}$ \\
\hline & Both-leg squat and ankle pump & $3 \times 10 \mathrm{r}$ \\
\hline & Single-leg stance with a swimming board & $2 \times 20 s$ \\
\hline & $\begin{array}{l}\text { Circle walking (clockwise and anti-clockwise, while one is standing on a single } \\
\text { foot in the center of circle) }\end{array}$ & $3 \times 30 s$ \\
\hline & Front-to-back weight shift & $3 \times 7 r$ \\
\hline & Axial rotation of the foot & $3 \times 6 r$ \\
\hline \multirow{6}{*}{4} & Straight walking (marching) & $2 \times 30 \mathrm{~m}$ \\
\hline & Static squat with shoulder flexion and extension & $3 \times 15 s$ \\
\hline & Single-leg stance without a swimming board & $2 \times 20 \mathrm{~s}$ \\
\hline & $\begin{array}{l}\text { Circle walking (clockwise and anti-clockwise, while one is standing on a single } \\
\text { foot in the center of circle and creating perturbations) }\end{array}$ & $3 \times 30 s$ \\
\hline & Side bending & $3 \times 7 r$ \\
\hline & Axial rotation of the foot (by increasing the size of circle) & $3 \times 6 r$ \\
\hline
\end{tabular}




\begin{tabular}{|c|c|c|}
\hline & Balance Exercises (30 Min) & \\
\hline Week & Exercises & Set $\times$ Repetition \\
\hline \multirow{6}{*}{5} & Straight walking by stopping on the resting leg & $2 \times 30 \mathrm{~m}$ \\
\hline & Static squat with toe and the circular rotation of the shoulder & $3 \times 15 \mathrm{~s}$ \\
\hline & Single-leg stance with eyes closed & $2 \times 20 s$ \\
\hline & $\begin{array}{l}\text { Circle walking (clockwise and anti-clockwise, while one is standing on a single } \\
\text { foot in the center of circle with eyes closed) }\end{array}$ & $3 \times 30 \mathrm{~s}$ \\
\hline & Side bending and creating perturbations & $3 \times 7 r$ \\
\hline & Both-leg forward and backward squat jump & $3 \times 8 r$ \\
\hline \multirow{6}{*}{6} & Leg scissors with switching leg & $3 \times 30 s$ \\
\hline & Static squat with toe and the circular rotation of shoulder & $3 \times 15 \mathrm{~s}$ \\
\hline & Single-leg stance with eyes closed & $3 \times 15 \mathrm{~s}$ \\
\hline & $\begin{array}{l}\text { Circle walking (clockwise and anti-clockwise, while one is standing on a single } \\
\text { foot in the center of circle with eyes closed) }\end{array}$ & $3 \times 30 \mathrm{~s}$ \\
\hline & Side bending and creating perturbations & $3 \times 7 r$ \\
\hline & Both-leg forward and backward squat jump & $3 \times 8 r$ \\
\hline
\end{tabular}

Cool Down (5 Min)

Breathing control movements
Perform the movements at the end of all training sessions as described

\begin{abstract}
Stretching movements

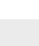

and concentration for the subjects while performing the task, soft cushions were placed around the test area.

To begin the test, the subject was asked to slightly move forward, land on the test foot, and maintain balance for 5 seconds. The subjects were taught to land on their toes and do not jump before landing. During the performance of the drop-landing task, the surface EMG device (Megawin model) recorded the activity of gluteus maximus, gluteus medius and rectus femoris muscles in the hip and lateral gastrocnemius, peroneus longus and tibialis anterior muscles in the ankle [25-27]. The muscle feedback activity was analyzed before initial ground contact until $250 \mathrm{~m} / \mathrm{s}$ after ground contact [28].
\end{abstract}

Holding for 30 seconds

PHYSICAL TREA TMENTS

In EMG signal processing, to compare different muscles and different subjects, muscle activity should be normalized to a reference value [28]. For this purpose, the values obtained from calculating the Root Mean Square (RMS) of muscle activity during perturbation were divided by the values obtained from the Maximum Voluntary Contraction (MVC) of each muscle. Furthermore, muscle activity was described as a percentage of maximum MVC (\%MVC). Each MVC was repeated twice for 5 seconds, and the mean score of the middle 3 seconds of MVC was used to normalize the obtained data [29].

After finishing the pretest phase of study, aquatic balance exercises were conducted in a shallow part of a covered indoor swimming pool at Shahid Beheshti University at a temperature of $26-28^{\circ} \mathrm{C}$ under the supervision of examiner (a an aquatic therapy coach). All the training exercises were first taught to the subjects by the examiner, then they were asked to perform practical trials [28]. The body weight is reduced to $40 \%$ $51 \%$ after Anterior Superior Iliac Spine (ASIS) level of immersion in the pool [30]. 
In the first week, the WV group performed the exercises without any additional weight and only by wearing vests. From the second week, with weight loss in the pool water, weights were added to the vests as 10 $20 \%$ of body weight. The design of vests allowed the symmetrical distribution of load on the lumbar spine and pelvis. The addition of weights was performed in a way to avoid flexor torque during exercise; the subjects could perform balance exercises in a safe environment $[13,15,31,32]$.

The exercises were performed for 6 weeks, 3 sessions per week, and each session lasted 40-45 minutes. First, there was a 10 -min warm up, then a 30 -min balance exercises in water, and eventually, a 5-min cool down [30, 33-35]. The exercises performed in the WV group started using a WV equal to $10 \%$ of their body weight and gradually completed by the end of the 6th week with a WV equal to $20 \%$ of the body weight [14]. The overload principle (FITT Principle) during the training sessions was gradually applied. With increasing intensity, using combined movements, the number of repetitions increased and the rest period between the sets reduced [36]. For the uniformity and possibility of comparing the effects of WVs, a fixed training protocol was designed for both study groups (Table 1). After completing the 6 weeks of aquatic, all of the aforementioned tests were performed at the post-test phase.
For analyzing data, we used descriptive and inferential statistics by SPSS. For testing the normality of data distribution, Shapiro-Wilk test; and for examining the interaction effect of time (before and after intervention) on the group (WV and control), Analysis of Covariance (ANCOVA) were used. The Cohen's d effect size for each between the two groups was also calculated where a size of 0.2 indicates a 'small' effect size, 0.5 a 'medium' effect size and 0.8 a 'large' effect size. In analyzing data, a $95 \%$ confidence level $(\mathrm{P} \leq 0.05)$ was considered.

\section{Results}

The demographic characteristics of subjects, including age, height, weight, and BMI are presented in Table 2. The Independent Samples t-test results indicated no significant difference between the two groups in terms of age, height, weight, and BMI. After confirming the normality of data distribution by Shapiro-Wilk test, ANCOVA was used to examine the effect of aquatic balance exercise with and without WV on balance recovery strategy (the ratio of hip muscle feedback activity to ankle muscle feedback activity) of the subjects. Table 3 presents the Mean \pm SD scores of feedback activity for selected hip and ankle muscles before and after intervention in both study groups.

ANCOVA results indicated a positive effect of aquatic balance exercise with WV on improving balance recov-

Table 2. The Independent Samples t-test results for comparing the demographic characteristics of participants (Mean \pm SD)

\begin{tabular}{cccc}
\hline Characteristics & WV & Control & Sig. \\
\hline Age $(\mathrm{yr})$ & $57.3 \pm 4.4$ & $57.7 \pm 5.4$ & 0.13 \\
Height $(\mathrm{cm})$ & $159 \pm 6.1$ & $161 \pm 5.6$ & 0.09 \\
Weight $(\mathrm{kg})$ & $65.3 \pm 4.5$ & $64.9 \pm 3.9$ & 0.1 \\
BMI $\left(\mathrm{kg} / \mathrm{m}^{2}\right)$ & $25.9 \pm 3.8$ & $25.4 \pm 4.4$ & 0.18 \\
\hline
\end{tabular}

Table 3. The Mean \pm SD scores of feedback activity for selected hip and ankle muscles and their ratio at the pretest and post-test phases

\begin{tabular}{cccccc}
\hline & & Pretest Muscle Activity & Hip-to-Ankle Ratio & Post-Test Muscle Activity & Hip-to-Ankle Ratio \\
\hline \multirow{2}{*}{ WV } & Hip joint & $65 \pm 7.8$ & 1.58 & $68 \pm 10.1$ & 1.15 \\
& Ankle joint & $41 \pm 4.9$ & & $59 \pm 8.4$ & $69 \pm 8.8$ \\
\multirow{2}{*}{ Control } & Hip joint & $67 \pm 6.3$ & 1.59 & $43 \pm 6.5$ & 1.56 \\
& Ankle joint & $42 \pm 11.3$ & & \\
\hline
\end{tabular}


ery strategy (transfer of strategy from hip to ankle) in middle-aged women $(\mathrm{F}=23.2, \mathrm{P}=0.003$, Eta $=0.76)$. The Eta coefficient of 0.76 also indicates a relatively large effect size of the intervention.

\section{Discussion}

The present study evaluated the effect of a 6-week aquatic balance exercise program with and without a $\mathrm{WV}$ on balance the recovery strategy of middle-aged women. The obtained results revealed that this training technique can significantly improve the balance recovery strategy $(\mathrm{P}<0.05)$ and reduce the hip-to-ankle ratio.

A distinctive feature of aquatic balance exercises used in this study, compared to the other methods of aquatic therapy exercises is the use of additional weight by wearing a vest when exercising in water. Most of the studies conducted on balance and its recovery strategies have reported the relationship between the reduction of ankle muscle strength and the switch of balance recovery strategy from ankle to hip $[9,12,18]$ Kamrani Faraz et al. [37] in line with the findings of Maeda et al. [38], stated that the weakness of dorsi- and plantar flexion muscles of ankle caused by aging and immobility can transfer the balance recovery strategy from ankle to hip and increase the risk of falling at old age [39]. The use of WVs in this study for balance exercises probably improved the muscle strength of the ankle joint, plantar sensory inputs and the activity of ankle muscles when applying perturbations. It also switched the balance recovery strategy from hip to ankle [19].

Most of the studies that have used aquatic exercises to improve balance in different communities have only considered static and dynamic balance indices, and recovery strategy as the most important factor in maintaining and recovering balance. The most important cause of falling in individuals has received less attention [30, 33-35]. Beyranvand et al. reported no effect of aquatic exercises on improving the balance strategy in elderly men; only the static and dynamic balance of elderly men improved after an 8 weeks of aquatic exercises, in their research [21]. In our study, the subjects were middleaged menopausal women with recent extensive physiological changes; while in Beyranvand et al.'s study, samples were elderly men.

The difference in subjects' age and gender as well as the training exercises can also be other reasons for the difference between the results of this study and those of Beyranvand and colleagues. They used commonly applied aquatic exercises in other studies; however, in the current study included the addition of external load by wearing vests. We also considered a combination of factors affecting balance such as strength, speed, agility and challenging variables of proprioception sense in lower limb joints [30, 33-35].

Using extra load during training can increase postexercise neuromuscular adaptation. This increase in neuromuscular adaptations cause the use of more effective neural units, the facilitation of movements of fastcontraction and large motor units, the increased coordination of muscles, reorganization in the sensory cortex, the increased efficiency and power of synaptic communication, the increased activation of nervous system, the reduction of inhibitory neural reflexes, the reduced resistance of neural pathways to impulse transmission, and the facilitation of sensory transfer inputs $[12,40]$. In addition, performing resistance training exercises inside a safe environment (water) can increase the strength of lower extremity muscles and muscle coordination.

Water is a safe and fun environment for exercising, compared to dry land, and encourages people to exercise and have physical activity, especially in older ages [40]. Improved balance recovery and control by aquatic activities are the consequences of the fact that water allows individuals to carry out a wide range of movements without the fear of being injured. Furthermore, the disruptive forces of stability provide an appropriate environment for balance exercises and challenging the systems involved in balance, including the system for sending afferent signals and returning efferent signals which can be effective in improving the body posture control [40].

The continuous challenge of balance in water environment due to the presence of water currents. In particular, wearing a WV and getting more disturbed activate proprioceptive receptors in the lower limb joints. Thus, this process continuously maintains the cycle of data transmission to the central and peripheral nervous systems. Subsequently, it sends afferent feedbacks to the lower limb muscles to correct motion errors.

This is repeated during a 6-week aquatic exercise program with a WV, which eventually corrects motor errors. Therefore, there is no need for processing in the central nervous system for transferring information and increasing the time for sending muscle afferent feedbacks; the peripheral nervous system automatically maintains and recovers the balance [41]. In this regard, improved balance recovery strategy after 6-week aquatic exercises 
with a WV is also probably due to the increased neuromuscular system efficiency.

This is because the continuous involvement of lower limb muscle feedback activity has probably led to the retraining of motor error correcting systems and the feedforward activation of muscles in this region. This indicates that after aquatic exercises, the ankle muscles are trained to have the readiness to cope with perturbations before facing the stimulus, control them at ankle joint level, and prevent them from transmission to the higher joint levels in lower extremities, from the transfer of the center of gravity, the base of support, and finally from falling.

Exploring the feedforward activity of lower limb muscles was not among the main purposes of this study. However, the information obtained from the surface EMG device demonstrated improvement in feedforward muscle activity; this data suggests the improved functioning of motor error correction system in the neuromuscular system [41]. An advantage of the present study, compared to other studies was the tool used to assess the balance recovery strategy. Previous studies have reported that the most reliable tool for assessing the balance recovery strategy is the EMG device, and examining muscle activity when faced with an external perturbation.

Aquatic exercises with a WV can be effective in transferring balance recovery strategy from the hip to ankle in middle-aged women who are entering old age and facing musculoskeletal problems. Obviously, this reduces the risk of falling when faced with a sudden perturbation. The present study considered the exercises that cover all factors affecting balance such as strength, proprioception sense, speed, agility and response time. Thus, it is suggested that sport rehabilitators, especially those treating middle-aged people use the proposed balance exercises to better prepare them for coping with aging.

\section{Ethical Considerations}

\section{Compliance with ethical guidelines}

This study was approved by the Research Ethics Committee of Shahid Beheshti University (code: IR.SBU. ICBS.97.1006)

\section{Funding}

The present paper was extracted from the MSc. thesis of the first author, in Department of Health and Sport
Sciences, Faculty of Sport Sciences and Health, Shahid Beheshti University.

\section{Authors' contributions}

Conceptualization, Investigation, Funding acquisition, Resources: All authors; Methodology: Niloufar Rahmani and Amirhossein Barati; Writing-original draft: Niloufar Rahmani; Writing, reviewing and editing: Amirhossein Barati and Fariborz Hovanloo; and Supervision: Amirhossein Barati.

\section{Conflict of interest}

The authors declared no conflict of interest.

\section{References}

[1] Movahed M, Hoseini M. Exploring the middle-aged women's strategies to deal with aging and age-related physical changes. Quarterly Journal of Women's Studies Sociological and Psychological. 2017; 14(4):7-42. [DOI:10.22051/ JWSPS.2017.2673]

[2] Statistical Center of Iran. [Selected results of the General Census of Population and Housing (Persian)]. Tehran: Statistical Center of Iran; 2016.

[3] Ambrose AF, Paul G, Hausdorff JM. Risk factors for falls among older adults: A review of the literature. Maturitas. 2013; 75(1):51-61. [DOI:10.1016/j.maturitas.2013.02.009] [PMID]

[4] Chiviacowsky S, Wulf G, Wally R. An external focus of attention enhances balance learning in older adults. Gait \& Posture. 2010; 32(4):572-5. [DOI:10.1016/j.gaitpost.2010.08.004] [PMID]

[5] Jorgensen M, Rathleff MS, Laessoe U, Caserotti P, Nielsen O, Aagaard P. Time-of-day influences postural balance in older adults. Gait \& Posture. 2012; 35(4):653-7. [DOI:10.1016/j. gaitpost.2011.12.018] [PMID]

[6] Lord SR, Sherrington C, Menz HB, Close JC. Falls in older people: Risk factors and strategies for prevention. Cambridge: Cambridge University Press; 2007. [DOI:10.1017/ CBO9780511722233

[7] Soleimani T, Noorbakhsh M, Alijani E. [The effect of 12 weeks of aerobic exercise and exercise on water on the quality of life and happiness of non-athlete midwives (Persian)] Motor Behavior. 2012; 10(1):105-22.

[8] Lopes K, Costa D, Santos L, Castro D, Bastone A. Prevalence of fear of falling among a population of older adults and its correlation with mobility, dynamic balance, risk and history of falls. Brazilian Journal of Physical Therapy. 2009; 13(3):223-9. [DOI:10.1590/S1413-35552009005000026]

[9] Beyranvand R, Sahebozamani M, Daneshjoo A. [A survey on relationship between postural sway parameters and balance 
recovery strategies in older people (Persian)]. Daneshvar Medicine. 2016; 23(123):21-30.

[10] Amiridis IG, Hatzitaki V, Arabatzi F. Age-induced modifications of static postural control in humans. Neuroscience Letters. 2003; 350(3):137-40. [DOI:10.1016/S03043940(03)00878-4]

[11] Mackey DC, Robinovitch SN. Mechanisms underlying agerelated differences in ability to recover balance with the ankle strategy. Gait \& Posture. 2006; 23(1):59-68. [DOI:10.1016/j. gaitpost.2004.11.009] [PMID]

[12] Beyranvand R, Sahebozamani M, Daneshjoo A, Seyedjafari E. [Relationship between changes in muscle strength and postural sway after eight weeks aquatic exercise in elderly people: A clinical trial (Persian)]. Journal of Mazandaran University of Medical Sciences. 2018; 27(157):92-104.

[13] Roghani T, Torkaman G, Movasseghe Sh, Hedayati M, Goosheh B, Bayat N. [The effect of 6-week submaximal training with and without external loading on cardiovascular fitness, balance, cortisol, and lipid profiles in osteoporotic postmenopausal women (Persian)]. Iranian Journal of Endocrinology and Metabolism. 2012; 13(6):587-97.

[14] Bean JF, Herman S, Kiely DK, Frey IC, Leveille SG, Fielding RA, et al. Increased Velocity Exercise Specific to Task (InVEST) training: A pilot study exploring effects on leg power, balance, and mobility in community-dwelling older women Journal of the American Geriatrics Society. 2004; 52(5):799804. [DOI:10.1111/j.1532-5415.2004.52222.x] [PMID]

[15] Shaw JM, Snow CM. Weighted vest exercise improves indices of fall risk in older women. The Journals of Gerontology Series A: Biological Sciences and Medical Sciences. 1998; 53(1):M53-M8. [DOI:10.1093/gerona/53A.1.M53] [PMID]

[16] Taheri M. Effect of hydrotherapy on lower body strength and balance among elderly women. Journal of Physical Education Research. 2015; 2(4):19-26.

[17] Vanaky B, Sadeghi H, Piri M, Ramezani N. [The effect of weight bearing water aerobic exercise on the bone density of the lumbar spine of 50-70 years old overweight women (Persian)]. Scientific Journal of Rehabilitation Medicine. 2015; 4(2):46-52.

[18] Sahebozamani M, Beyranvand R, Ebrahimipour E, Razavi $M$. The effect of 8 weeks deep-aquatic exercises on static balance and lower body strength among elderly men. International Journal of Applied Exercise Physiology. 2017; 6(1):8698. [DOI:10.22631/ijaep.v6i1.115]

[19] Rezvani MH, Rahmani Gh. [The effect of eight weeks of Palett exercises on the static and dynamic balance of people with stroke (Persian)]. Journal of Research in Sport Rehabilitation. $2015 ; 3(6): 11-9$.

[20] Chow SC, Shao J, Wang H, Lokhnygina Y. Sample size calculations in clinical research. Boca Raton, Florida: Chapman and Hall/CRC; 2017. [DOI:10.1201/9781315183084]

[21] Holviala JH, Sallinen JM, Kraemer WJ, Alen MJ, HÄkkinen KK. Effects of strength training on muscle strength characteristics, functional capabilities, and balance in middle-aged and older women. Journal of Strength and Conditioning Research. 2006; 20(2):336-44. [DOI:10.1519/00124278200605000-00018] [PMID]
[22] Nagano Y, Ida H, Akai M, Fukubayashi T. Gender differences in knee kinematics and muscle activity during single limb drop landing. The Knee. 2007; 14(3):218-23. [DOI:10.1016/j.knee.2006.11.008] [PMID]

[23] Morey-Klapsing G, Arampatzis A, Brüggemann GP. Choosing EMG parameters: Comparison of different onset determination algorithms and EMG integrals in a joint stability study. Clinical Biomechanics. 2004; 19(2):196-201. [DOI:10.1016/j.clinbiomech.2003.10.010] [PMID]

[24] Hermens HJ, Freriks B, Merletti R, Stegeman D, Blok J, Rau $\mathrm{G}$, et al. European recommendations for surface electromyography. Enschede: Roessingh Research and Development; 1999.

[25] Rezaiee K, Mazloum V, Mamashli A. [A Comparison of Timing of Core Muscles between ACL Reconstructed and Healthy Athletes While Landing (Persian)]. Sport Medicine. 2016; 8(16):35-51.

[26] Rietdyk S, Patla A, Winter D, Ishac M, Little C. Balance recovery from medio-lateral perturbations of the upper body during standing. Journal of Biomechanics. 1999; 32(11):114958. [DOI:10.1016/S0021-9290(99)00116-5]

[27] Murillo DB, Solana RS, Vera-Garcia FJ, Fuertes NG, Moreno FJ. Effect of increasing difficulty in standing balance tasks with visual feedback on postural sway and EMG: Complexity and performance. Human Movement Science. 2012 31(5):1224-37. [DOI:10.1016/j.humov.2012.01.002] [PMID]

[28] Kalantariyan M, Minoonejad H, Rajabi R, Beyranvand R, Zahiri A. [The comparison of the electromyography activity of selected muscles of the ankle joint in athletes with ankle dorsiflexion range of motion limitation with healthy athletes during the single-leg jump landing (Persian)]. Scientific Journal of Rehabilitation Medicine. 2013; 2(2):14-23.

[29] Health UDo, Services H. Selected topics in surface electromyography for use in the occupational setting: Expert perspectives. Atlanta: Centers for Disease Control and Prevention; 1992.

[30] Kargarfard M, Shariat A, Ingle L, Cleland JA, Kargarfard M. Randomized controlled trial to examine the impact of aquatic exercise training on functional capacity, balance, and perceptions of fatigue in female patients with Multiple Sclerosis. Archives of Physical Medicine and Rehabilitation. 2018; 99(2):234-41. [PMID]

[31] Harrison R, Bulstrode S. Percentage weight-bearing during partial immersion in the hydrotherapy pool. Physiotherapy Practice. 1987; 3(2):60-3. [DOI:10.3109/09593988709087741]

[32] Prentice WE, Kaminski TW. Rehabilitation techniques for sports medicine and athletic training. New York: McGrawHill; 2004.

[33] Layne M. Water Exercise. Champaign, Illinois: Human Kinetics; 2015.

[34] Resende SM, Rassi CM. Effects of hydrotherapy in balance and prevention of falls among elderly women. Brazilian Journal of Physical Therapy. 2008; 12(1):57-63. [DOI:10.1590/ S1413-35552008000100011

[35] Fisken AL, Waters DL, Hing WA, Steele M, Keogh JW. Comparative effects of 2 aqua exercise programs on physical function, balance, and perceived quality of life in older adults with osteoarthritis. Journal of Geriatric Physical Ther- 
apy. 2015; 38(1):17-27. [DOI:10.1519/JPT.0000000000000019] [PMID]

[36] Sarhadi S, Ramezani A, Gholami M, Taheri H. The effect of an aerobic training cycle in the morning and evening on primary dysmenorrhea and some physiological variables in matured girls (Persian)]. Iranian Journal of Obstetrics, Gynecology and Infertility. 2015; 18(162):11-20.

[37] Kamrani Faraz N, Letafat Kar A, Javdaneh N. [Effect of a water resistance combined training on balance and fall risk in elderly women over 60 years in Tehran (Persian)]. Iranian Journal of Rehabilitation Research in Nursing. 2017; 4(1):4551.

[38] Maeda Y, Tanaka T, Nakajima Y, Miyasaka T, Izumi T, Kato $\mathrm{N}$. Age-related changes in dynamic postural control ability in the presence of sensory perturbation. Journal of Medical and Biological Engineering. 2015; 35(1):86-93. [DOI:10.1007/ s40846-015-0009-8]

[39] Douris P, Southard V, Varga C, Schauss W, Gennaro C, Reiss A. The effect of land and aquatic exercise on balance scores in older adults. Journal of Geriatric Physical Therapy. 2003; 26(1):3-6. [DOI:10.1519/00139143-200304000-00001]

[40] Arabameri E, Taheri M, Irandoust Kh. [The effect of water-based exercise programs and KSD protocol on general health questionnaire and optimal physical fitness factors affecting female elders balance (Persian)]. Motor Behavior. 2014; 16:15-28.

[41] Letafatkar A, Rajabi R, Ebrahimi Tekamejani E, Minoonejad $\mathrm{H}$. [The effect of perturbation training on timing of hamstring and quadriceps electromyography activation in active subjects with neuromuscular dominance quadriceps deficit (Persian)]. Sport Physiology \& Management Investigations. 2015; 6(4):469-81. 